\title{
CENTERS AND ISOCHRONOUS CENTERS FOR TWO CLASSES OF GENERALIZED SEVENTH AND NINTH SYSTEMS
}

\author{
JAUME LLIBRE ${ }^{1}$ AND CLÀUDIA VALLS ${ }^{2}$
}

\begin{abstract}
We classify new classes of centers and of isochronous centers for polynomial differential systems in $\mathbb{R}^{2}$ of arbitrary odd degree $d \geq 7$ that in complex notation $z=x+i y$ can be written as

$\dot{z}=(\lambda+i) z+(z \bar{z})^{\frac{d-7-2 j}{2}}\left(A z^{5+j} \bar{z}^{2+j}+B z^{4+j} \bar{z}^{3+j}+C z^{3+j} \bar{z}^{4+j}+D \bar{z}^{7+2 j}\right)$, where $j$ is either 0 or $1, \lambda \in \mathbb{R}$ and $A, B, C \in \mathbb{C}$. Note that if $j=0$ and $d=7$ we obtain a special case of seventh polynomial differential systems which can have a center at the origin, and if $j=1$ and $d=9$ we obtain a special case of ninth polynomial differential systems which can have a center at the origin.
\end{abstract}

\section{Introduction AND STATEMENT OF THE MAIN RESUlts}

In this paper we consider the polynomial differential systems in the real $(x, y)$-plane that has a singular point at the origin with eigenvalues $\lambda \pm i$ and that can be written as

(1) $\dot{z}=(\lambda+i) z+(z \bar{z})^{\frac{d-7-2 j}{2}}\left(A z^{5+j} \bar{z}^{2+j}+B z^{4+j} \bar{z}^{3+j}+C z^{3+j} \bar{z}^{4+j}+D \bar{z}^{7+2 j}\right)$,

where $j$ is either 0 or $1, z=x+i y, d \geq 7$ is an arbitrary odd positive integer, $\lambda \in \mathbb{R}$ and $A, B, C \in \mathbb{C}$. When $j=0$ we are considering the class of systems

$$
\dot{z}=(\lambda+i) z+(z \bar{z})^{\frac{d-7}{2}}\left(A z^{5} \bar{z}^{2}+B z^{4} \bar{z}^{3}+C z^{3} \bar{z}^{4}+D \bar{z}^{7}\right),
$$

while when $j=1$ we are considering the class of systems

$$
\dot{z}=(\lambda+i) z+(z \bar{z})^{\frac{d-9}{2}}\left(A z^{6} \bar{z}^{3}+B z^{5} \bar{z}^{4}+C z^{4} \bar{z}^{5}+D \bar{z}^{9}\right) .
$$

The vector field associated to this system is formed by the linear part $(\lambda+i) z$ and by a homogeneous polynomial of degree $d$ formed by four monomials in complex notation. The origin is either a weak focus or a center if $\lambda=0$, see for instance $[1,15]$.

For such systems we want to determine the conditions that ensure that the origin of (1) is a center or an isochronous center. Of course these systems for $j=0$ and $d=7$ coincide with a class of seventh polynomial differential systems. So we call the class of polynomial differential systems (1) of odd degree $d \geq 7$ the generalized seventh systems. When $j=1$ and $d=9$ these systems coincide with a class of ninth polynomial differential systems. So

\footnotetext{
Key words and phrases. centers, isochronous centers, polynomial vector fields.
} 
we call the class of polynomial differential systems (1) of odd degree $d \geq 9$ the generalized ninth systems.

The problem of characterizing the centers and the isochronous centers has attracted the attention of many authors. However there are very few families of polynomial differential systems in which a complete classification of centers or of isochronous centers has been done. Quadratic systems were classified by Dulac in [6], Kapteyn in [10, 11], Bautin in [2], Żołądek in [19], and Loud in [14], while the cubic systems with homogeneous nonlinearities were classified by $[13,18,20]$. But we are very far to obtain a complete classification of the centers for the polynomial differential systems of degree 3. As far as we know there were no results about centers and isochronous centers for degrees 7 or 9 .

The main result in this paper is the following one, which provides families of centers and of isochronous centers of arbitrary high degree.

Theorem 1. For $d \geq 7+2 j$ odd the following statements hold for system (1).

(a) It has a center at the origin if and only if one of the following three conditions holds.

(a.1) $\lambda=b_{1}=(5+j) A+(3+j) \bar{C}=0$ if $j=0,1$,

(a.2) $\lambda=b_{1}=\operatorname{Im}(A C)=\operatorname{Re}\left(A^{4} D\right)=\operatorname{Re}\left(\bar{C}^{4} D\right)=0$ if $j=0$,

(a.3) $\lambda=b_{1}=\operatorname{Im}(A C)=\operatorname{Im}\left(A^{5} D\right)=\operatorname{Im}\left(\bar{C}^{5} D\right)=0$ if $j=1$.

Note that when condition (a.1) holds we have a Hamiltonian center and when either condition (a.2) or condition (a.3) holds we have a reversible center.

(b) It has an isochronous center at the origin if and only if one of the following two conditions holds.

(b.1) $\lambda=B=D=0, C=\bar{A}$,

(b.2) $\lambda=B=D=0, C=(3-d) \bar{A} /(d+1)$.

Note that if in Theorem 1 condition (a.1) holds and $D=0$, then we have a special center that also satisfies either condition (a.2) if $j=0$, or condition (a.3) if $j=1$. This is a special center that is simultaneously Hamiltonian and reversible.

The families of centers obtained in Theorem 1 were not known. Moreover the families of isochronous centers were not known till now because they did not appear in the well-known survey on the isochronous centers [3].

The paper is devoted to the proof of Theorem 1. To do it we have divided the paper as follows. In Section 2 we introduce some preliminaries that will be used later on. In Section 3 we provide the proof of Theorem 1(a), while the proof of Theorem 1(b) is given in Section 4. 


\section{Preliminaries}

The proof of Theorem 1 needs the effective computation of the Liapunov constants as well as of the period constants. We write

$$
A=a_{1}+i a_{2}, \quad B=b_{1}+i b_{2}, \quad C=c_{1}+i c_{2}, \quad D=d_{1}+i d_{2} .
$$

Writing system (1) in polar coordinates, i.e, doing the change of variables $r^{2}=z \bar{z}$ and $\theta=\arctan (\operatorname{Im} z / \operatorname{Re} z)$, it becomes

$$
\frac{d r}{d \theta}=\frac{\lambda r+F(\theta) r^{d}}{1+G(\theta) r^{d-1}}
$$

where

$$
\begin{gathered}
F(\theta)=\left(a_{1}+c_{1}\right) \cos (2 \theta)-\left(a_{2}-c_{2}\right) \sin (2 \theta)+b_{1}+ \\
d_{1} \cos ((8+2 j) \theta)+d_{2} \sin ((8+2 j) \theta), \\
G(\theta)=\left(a_{2}+c_{2}\right) \cos (2 \theta)+\left(a_{1}-c_{1}\right) \sin (2 \theta)+b_{2} \\
+d_{2} \cos ((8+2 j) \theta)-d_{1} \sin ((8+2 j) \theta) .
\end{gathered}
$$

Since $\dot{\theta}=1+G(\theta) r^{d-1}$, sufficiently close to the origin $\dot{\theta}>0$. So if system (1) has a center at the origin the same occurs for system (2).

The transformation $(r, \theta) \mapsto(\rho, \theta)$ defined by

$$
\rho=\frac{r^{d-1}}{1+G(\theta) r^{d-1}}
$$

is a diffeomorphism from the region $\dot{\theta}>0$ into its image. If we write equation

(2) in the variable $\rho$, we obtain the following Abel differential equation

$$
\begin{aligned}
\frac{d \rho}{d \theta}= & (d-1) G(\theta)[\lambda G(\theta)-F(\theta)] \rho^{3}+ \\
& {\left[(d-1)(F(\theta)-2 \lambda G(\theta))-G^{\prime}(\theta)\right] \rho^{2}+(d-1) \lambda \rho } \\
= & A(\theta) \rho^{3}+B(\theta) \rho^{2}+C \rho .
\end{aligned}
$$

These kind of differential equations appeared in the studies of Abel on the theory of elliptic functions. For more details on Abel differential equations, see [9], [4] or [8].

The solution $\rho(\theta, \gamma)$ of (4) satisfying that $\rho(0, \gamma)=\gamma$ can be expanded in a convergent power series of $\gamma \geq 0$ sufficiently small. Thus

$$
\rho(\theta, \gamma)=\rho_{1}(\theta) \gamma+\rho_{2}(\theta) \gamma^{2}+\rho_{3}(\theta) \gamma^{3}+\ldots
$$

with $\rho_{1}(\theta)=1$ and $\rho_{k}(0)=0$ for $k \geq 2$. Let $P:\left[0, \gamma_{0}\right] \rightarrow \mathbb{R}$ be the Poincaré map defined by $P(\gamma)=\rho(2 \pi, \gamma)$ and for a convenient $\gamma_{0}>0$. Then the values of $\rho_{k}(2 \pi)$ for $k \geq 2$ controle the behavior of the Poincaré map in a neighborhood of $\rho=0$. Clearly system (1) has a center at the origin if and only if $\rho_{1}(2 \pi)=1$ and $\rho_{k}(2 \pi)=0$ for every $k \geq 2$. Assuming that $\rho_{2}(2 \pi)=\cdots=\rho_{m-1}(2 \pi)=0$ we say that $v_{m}=\rho_{m}(2 \pi)$ is the $m$-th Liapunov or Liapunov-Abel constant of system (1). These constants were also considered in the paper of Gasull, Guillamon and Mañosa [7]. 
The set of coefficients for which all the Liapunov constants vanish is called the center variety of the family of polynomial differential systems. By the Hilbert Basis Theorem the center variety is an algebraic set. Necessary conditions to have a center at the origin will be obtained by finding the zeros of the Liapunov constants.

We note that the centre manifold, i.e., the space of systems (1) with a centre at the origin is invariant with respect to the action group $C^{*}$ of changes of variables $z \rightarrow \xi z$ :

(6)

$$
\begin{array}{ll}
A \rightarrow \xi^{(d-9-2 j) / 2} \bar{\xi}^{(d-7-2 j) / 2} \xi^{5+j} \bar{\xi}^{2+j} A, & B \rightarrow \xi^{(d-9-2 j) / 2} \bar{\xi}^{(d-7-2 j) / 2} \xi^{4+j} \bar{\xi}^{3+j} B, \\
C \rightarrow \xi^{(d-9-2 j) / 2} \bar{\xi}^{(d-7-2 j) / 2} \xi^{3+j} \bar{\xi}^{4+j} C, & D \rightarrow \xi^{(d-9-2 j) / 2} \bar{\xi}^{(d-7-2 j) / 2} \bar{\xi}^{7+2 j} D .
\end{array}
$$

To show the sufficiency of the found conditions we look for the existence of a local analytic first integral defined in a neighborhood of the origin, or we will show that system (1) is reversible. We recall that system (1) is reversible with respect to a straight line if it is invariant under the change of variables $\bar{w}=e^{i \gamma} z, \tau=-t$ for some $\gamma$ real. For system (1) we have the following result whose proof can be found in [5].

Lemma 2. System (1) is reversible if and only if $A=-\bar{A} e^{2 i \gamma}, B=-\bar{B}$, $C=-\bar{C} e^{-2 i \gamma}$ and $D=-\bar{D} e^{-(8+2 j) i \gamma}$ for some $\gamma \in \mathbb{R}$. Furthermore in this situation the origin of system (1) is a center.

Once we have proven the existence of the so-called center variety of system (1) we also want to compute which of the centers are isochronous. In that case, let $z=0$ be a center (that is, we assume that we are under the hypothesis that guarantee that $z=0$ is a center) and let $V$ be a neighborhood of $z=0$ covered with periodic orbits surrounding $z=0$. We can define a function, the period function of $z=0$ by associating to every point $z$ of $V$ the minimal period of the periodic orbit passing through $z$. The center $z=0$ of system (1) is isochronous if the period of all integral curves in $V \backslash\{0\}$ is constant.

If we take the equation of $\theta^{\prime}=d \theta / d t$ and we apply the change of variables in (2) we obtain

$$
\begin{aligned}
T & =\int_{0}^{2 \pi} \frac{d \theta}{\theta^{\prime}}=\int_{0}^{2 \pi} \frac{1}{1+G(\theta) r(\theta)^{d-1}} d \theta \\
& =\int_{0}^{2 \pi}(1-G(\theta) \rho(\theta)) d \theta=2 \pi-\int_{0}^{2 \pi} G(\theta) \rho(\theta) d \theta,
\end{aligned}
$$

where $\rho(\theta)=\sum_{j \geq 1} \rho_{j}(\theta) \gamma^{j}$ is given in $(5)$ and $\rho_{j}(\theta)$ are the terms giving rise to the Liapunov-Abel constants $\rho_{j}(2 \pi)$. Then system (1) has an isochronous center at the origin if it is a center and satisfies

$$
\int_{0}^{2 \pi} G(\theta) \varrho(\theta) d \theta=\sum_{j \geq 1}\left(\int_{0}^{2 \pi} G(\theta) \rho_{j}(\theta) d \theta\right) \gamma^{j}=0,
$$


that is, if

$$
T=\int_{0}^{2 \pi} \frac{d \theta}{\theta^{\prime}}=2 \pi-\sum_{j \geq 1} T_{j} \gamma^{j}=2 \pi,
$$

with

$$
T_{j}=\int_{0}^{2 \pi} G(\theta) \rho_{j}(\theta) d \theta=0, \quad \text { for } \quad j \geq 1 .
$$

The constants $T_{j}$ will be called the period Abel constants.

\section{Proof of Theorem 1(A)}

We divide the proof of Theorem 1(a) into different parts.

3.1. Sufficient conditions for a center. In this subsection we will see that conditions (a.1), (a.2) and (a.3) are sufficient to have a center at the origin. For this we will prove that system (1) under one of these conditions either has a first integral defined in a neighborhood of zero, or is reversible.

Under conditions (a.1) if we rescale system (1) by $|z|^{d-7-2 j}$ it becomes

$$
\begin{aligned}
\dot{z} & =i z|z|^{7+2 j-d}+A z^{5+j} \bar{z}^{2+j}+i b_{2} z^{4+j} \bar{z}^{3+j}-\frac{(5+j) \bar{A}}{3+j} z^{3+j} \bar{z}^{4+j}+D \bar{z}^{7+2 j} \\
& =i \frac{\partial H}{\partial \bar{z}}
\end{aligned}
$$

where for $d \geq 7+2 j$ odd we have

$$
\begin{aligned}
H= & \frac{2}{9+2 j-d}|z|^{9+2 j-d}-i \frac{A}{3+j} z^{5+j} \bar{z}^{3+j}+\frac{b_{2}}{4+j} z^{4+j} \bar{z}^{4+j}+i \frac{\bar{A}}{3+j} z^{3+j} \bar{z}^{5+j} \\
& -\frac{i}{8+2 j}\left(D \bar{z}^{8+2 j}-\bar{D} z^{8+2 j}\right) \quad \text { for } d \neq 9+2 j
\end{aligned}
$$

and

$$
\begin{aligned}
H= & \log |z|^{2}-i \frac{A}{3+j} z^{5+j} \bar{z}^{3+j}+\frac{b_{2}}{4+j} z^{4+j} \bar{z}^{4+j}+i \frac{\bar{A}}{3+j} z^{3+j} \bar{z}^{5+j} \\
& -\frac{i}{8+2 j}\left(D \bar{z}^{8+2 j}-\bar{D} z^{8+2 j}\right) \quad \text { for } d=9+2 j .
\end{aligned}
$$

Note that the integral $\exp (H)$ for $d=9+2 j$ and $H$ for $d \geq 7+2 j$ odd (with $d \neq 9+2 j$ ), are real and well defined at the origin. Therefore the origin is a Hamiltonian center.

From conditions (a.2) and (a.3), we have that (9)

$$
B=-\bar{B}, \quad \frac{\bar{A}}{A}=\frac{C}{\bar{C}}, \quad\left(\frac{\bar{A}}{A}\right)^{4+j}=(-1)^{1+j}\left(\frac{D}{\bar{D}}\right), \quad\left(\frac{\bar{C}}{C}\right)^{4+j}=(-1)^{1+j}\left(\frac{\bar{D}}{D}\right) .
$$


Now let $\theta_{1}, \theta_{2}$ and $\theta_{3}$ such that $e^{i \theta_{1}}=-\bar{A} / A, e^{i \theta_{2}}=-\bar{C} / C$ and $e^{i \theta_{3}}=$ $-\bar{D} / D$. Then by (9) we obtain

$$
\theta_{1}=-\theta_{2}(\bmod .2 \pi) \text { and } \theta_{2}=\frac{1}{4+j} \theta_{3}(\bmod .2 \pi) .
$$

Now take $\gamma=-\theta_{1} / 2$. Using (10) we have

and

$$
e^{2 i \gamma}=e^{-i \theta_{1}}=-\frac{A}{\bar{A}}, \quad e^{-2 i \gamma}=e^{i \theta_{1}}=e^{-i \theta_{2}}=-\frac{C}{\bar{C}},
$$

$$
e^{-(8+2 j) i \gamma}=e^{i(4+j) \theta_{1}}=e^{-i(4+j) \theta_{2}}=e^{-i \theta_{3}}=-\frac{D}{\bar{D}},
$$

which clearly implies that system (1) under condition (a.2), or (a.3) is reversible and thus it has a center at the origin.

3.2. Necessary conditions for a center. In this subsection we will see that conditions (a.1), (a.2) and (a.3) are necessary to have a center at the origin. For this we first compute the Liapunov constants up to some order and then show that the zeros of those Liapunov constants provide the conditions either (a.1), or (a.2), or (a.3).

Proposition 3. Let $j=0$. The Liapunov constants of system (1), with $d \geq 7$ odd, are

$$
\begin{aligned}
V_{1}= & e^{2 \pi(d-1) \lambda} \\
V_{2}= & b_{1} \\
V_{3}= & -\operatorname{Im}(A C), \\
V_{4}= & 0 \\
V_{5}= & 0 \\
V_{6}= & \operatorname{Re}((5 A+3 \bar{C}) D[(d-4) A+(d+2) \bar{C}][(d-9) A+(d+7) \bar{C}] \\
& \quad[(3 d-7) A+(3 d+1) \bar{C}]), \\
V_{7}= & \operatorname{Im}\left(( 5 A + 3 \overline { C } ) B D [ A - \overline { C } ] \left[\left(55 d^{2}-400 d+657\right) A^{2}\right.\right. \\
& \left.\left.\quad+\left(110 d^{2}-220 d-514\right) A \bar{C}+\left(55 d^{2}+180 d+77\right) \bar{C}^{2}\right]\right),
\end{aligned}
$$

We remark that $V_{k} \equiv \rho_{k}(2 \pi)\left(\bmod .\left\{V_{1}, V_{2}, \ldots, V_{k-1}\right\}\right)$ for $k=1, \ldots, 8$ and also modulo a positive constant.

Proof. Solving $\rho_{1}^{\prime}(\theta)=(d-1) \lambda \rho_{1}(\theta)$ and evaluating at $\theta=2 \pi$ we obtain $v_{1}=\rho_{1}(2 \pi)=e^{2 \pi(d-1) \lambda}$. Then $V_{1}=e^{2 \pi(d-1) \lambda}$. In order to have a center at the origin $\rho_{1}(2 \pi)=1$, so in what follows we take $\lambda=0$.

Substituting (5) into (4) we get that the functions $\rho_{k}(\theta)$ must satisfy

$$
\begin{aligned}
& \rho_{2}^{\prime}=B \rho_{1}^{2}, \\
& \rho_{3}^{\prime}=A \rho_{1}^{3}+2 B \rho_{1} \rho_{2}, \\
& \rho_{4}^{\prime}=3 A \rho_{1}^{2} \rho_{2}+B\left(\rho_{2}^{2}+2 \rho_{1} \rho_{3}\right), \\
& \rho_{5}^{\prime}=3 A\left(\rho_{1} \rho_{2}^{2}+\rho_{1}^{2} \rho_{3}\right)+2 B\left(\rho_{2} \rho_{3}+\rho_{1} \rho_{4}\right), \\
& \rho_{6}^{\prime}=A\left(\rho_{2}^{3}+6 \rho_{1} \rho_{2} \rho_{3}+3 \rho_{1}^{2} \rho_{4}\right)+B\left(\rho_{3}^{2}+2 \rho_{2} \rho_{4}+2 \rho_{1} \rho_{5}\right), \\
& \rho_{7}^{\prime}=3 A\left(\rho_{2}^{2} \rho_{3}+\rho_{1} \rho_{3}^{2}+2 \rho_{1} \rho_{2} \rho_{4}+\rho_{1}^{2} \rho_{5}\right)+2 B\left(\rho_{3} \rho_{4}+\rho_{2} \rho_{5}+\rho_{1} \rho_{6}\right),
\end{aligned}
$$


where we have omitted that all the functions depend on $\theta$. Note that all these differential equations can be solved recursively doing an integral between 0 and $\theta$, and recalling that $\rho_{k}(0)=0$ for $k \geq 2$. We have done all the computations of this paper with the help of the algebraic manipulator mathematica. These computations are not difficult but sometimes are long and tedious.

Solving the equation $\rho_{2}^{\prime}=B \rho_{1}^{2}$ we get that $\rho_{2}(2 \pi)=2 \pi(d-1) b_{1}$. Then $V_{2}=b_{1}$. From now on we take $b_{1}=0$.

Now we compute the solution $\rho_{3}(\theta)$ of $\rho_{3}^{\prime}=A \rho_{1}^{3}+2 B \rho_{1} \rho_{2}$, and we get that $\rho_{3}(2 \pi)=2 \pi(1-d) \operatorname{Im}(A C)$. Then $V_{3}=-\operatorname{Im}(A C)$.

Computing the solution $\rho_{k}(\theta)$ for $k=4,5$ from the differential equation for $\rho_{k}(\theta)$, we get $\rho_{k}(\theta)$ and in particular we obtain that $V_{k}=0$, being $V_{k}$ equal to $\rho_{k}(2 \pi)$ when $\rho_{2}(2 \pi)=\rho_{3}(2 \pi)=0$ for $k=4,5$.

Solving the differential equation for $\rho_{6}(\theta)$ we get $\rho_{6}(\theta)$ and in particular we obtain from the expression of $v_{6}=\rho_{6}(2 \pi)$ the value of $V_{6}$ given in the statement of Proposition 3 modulo $\rho_{2}(2 \pi)=\rho_{3}(2 \pi)=0$ and a positive constant. More precisely we can check that if we multiply $v_{6}$ by $-768 /((d-$ 1) $\pi$ ) then

$$
\begin{aligned}
v_{6}= & V_{6}+2 V_{3}\left(\left(1551+783 d-687 d^{2}+81 d^{3}\right) a_{2}\left(a_{2} d_{2}-a_{1} d_{1}\right)+\right. \\
& \left(-354+860 d+142 d^{2}-72 d^{3}\right) d_{2}\left(a_{2} c_{2}+a_{1} c_{1}\right)+ \\
& \left(-181-117 d+85 d^{2}+21 d^{3}\right) c_{2}^{2} d_{2} \\
& \left(-517-261 d+229 d^{2}-27 d^{3}\right) d_{2} a_{1}^{2}+ \\
& \left(543+351 d-255 d^{2}-63 d^{3}\right) c_{1}\left(d_{2} c_{1}-c_{2} d_{1}\right)+ \\
& \left.\left(177-430 d-71 d^{2}+36 d^{3}\right) d_{1}\left(c_{2} a_{1}-5 a_{2} c_{1}\right)\right) .
\end{aligned}
$$

We compute the solution $\rho_{7}(\theta)$ from the differential equation for $\rho_{7}(\theta)$, we get $\rho_{7}(\theta)$, and in particular we obtain the expression for $v_{7}=\rho_{7}(2 \pi)$ given in the statement of Proposition 3 modulo $\rho_{2}(2 \pi)=\rho_{3}(2 \pi)=\rho_{6}(2 \pi)=0$ and a positive constant. The computation of $V_{7}$ is done in the same way as $V_{6}$. This completes the proof of the proposition.

Proposition 4. Let $j=0$. For $d \geq 7$ odd if $V_{1}=1, V_{k}=0$ for $k=2, \ldots, 7$, then one of the following conditions holds.

(a.1) $\lambda=b_{1}=5 A+3 \bar{C}=0$,

(a.2) $\lambda=b_{1}=\operatorname{Im}(A C)=\operatorname{Re}\left(A^{4} D\right)=\operatorname{Re}\left(\bar{C}^{4} D\right)=0$,

(c.3) $\lambda=B=C=0, d=9$ and $\operatorname{Re}\left(A^{4} D\right) \neq 0$,

(c.4) $\lambda=B=(d-9) A+(d+7) \bar{C}=0, d \neq 9$ and condition (a.2) does not hold,

(c.5) $\lambda=B=(d-4) A+(d+2) \bar{C}=0$ and condition (a.2) does not hold,

(c.6) $\lambda=B=(3 d-7) A+(3 d+1) \bar{C}=0$ and condition (a.2) does not hold. 
Proof. From the fact that $V_{1}=1$ we get that $\lambda=0$. The condition $V_{2}=0$ implies that $b_{1}=0$. Furthermore to do $V_{3}=0$ we will consider two different cases: $C=0$ and $C \neq 0$. In this last case we have that $A=\mu \bar{C}$ with $\mu \in \mathbb{R}$.

Case 1: $C=0$. Therefore

$$
V_{6}=5(d-4)(d-9)(3 d-7) \operatorname{Re}\left(A^{4} D\right) .
$$

In view of the factors of $V_{6}$ and since $d \geq 7$ odd, we need to consider two different subcases.

Subcase 1.1: $\operatorname{Re}\left(A^{4} D\right)=0$. Then we are under the hypotheses of condition (a.2).

Subcase 1.2: $\operatorname{Re}\left(A^{4} D\right) \neq 0$ and $d=9$. We have

$$
V_{7}=7560 \operatorname{Im}\left(B D A^{4}\right)=7560 b_{2} \operatorname{Re}\left(A^{4} D\right) .
$$

To have $V_{7}=0$ we must impose $b_{2}=0$, that is, $B=0$. In this case we are under the hypotheses of condition (c.3).

Case 2: $A=\mu \bar{C}, \mu \in \mathbb{R}$ and $C \neq 0$. Then

$V_{6}=(5 \mu+3)((d-4) \mu+d+2)((d-9) \mu+d+7)((3 d-7) \mu+3 d+1) \operatorname{Re}\left(\bar{C}^{4} D\right)$.

In view of the factors of $V_{6}$ we need to consider five different subcases.

Subcase 2.1: $\mu=-3 / 5$. So we are under the hypotheses of condition (a.1).

Subcase 2.2: $\operatorname{Re}\left(\bar{C}^{4} D\right)=0$. Therefore we are under the hypotheses of condition (a.2).

Subcase 2.3: $\mu=-(d+2) /(d-4), \operatorname{Re}\left(\bar{C}^{4} D\right) \neq 0$. Since $b_{1}=0$, we have

$$
V_{7}=-\frac{1008(d-1)^{3}(d+11)}{(d-4)^{4}} b_{2} \operatorname{Re}\left(\bar{C}^{4} D\right) .
$$

Then, since $d \geq 7$ odd, we get that $V_{7}=0$ if and only if $b_{2}=0$, that is $B=0$. In this case we are under the hypothesis of condition (c.5).

Subcase 2.4: $\mu=-(d+7) /(d-9), \operatorname{Re}\left(\bar{C}^{4} D\right) \neq 0$ and $d \neq 9$. Since $b_{1}=0$, we have

$$
V_{7}=\frac{24192(d-1)^{3}(d+31)}{(d-9)^{4}} b_{2} \operatorname{Re}\left(\bar{C}^{4} D\right) .
$$

Then, since $d \geq 7$ odd with $d \neq 9$, we get that $V_{7}=0$ if and only if $b_{2}=0$, that is $B=0$. In this case we are under the hypothesis of condition (c.4).

Subcase 2.5: $\mu=-(3 d+1) /(3 d-7), \operatorname{Re}\left(\bar{C}^{4} D\right) \neq 0$. Since $b_{1}=0$, we have

$$
V_{7}=\frac{9984(d-1)^{3}(13+3 d)}{(3 d-7)^{4}} b_{2} \operatorname{Re}\left(\bar{C}^{4} D\right) .
$$

Then, since $d \geq 7$ odd, we get that $V_{7}=0$ if and only if $b_{2}=0$, that is $B=0$. In this case we are under the hypothesis of condition (c.6). This completes the proof of Proposition 4. 
Now we show that conditions (c.k) with $k=3, \ldots, 6$ do not provide a center at the origin.

Proposition 5. Let $j=0$. Condition (c.3) does not provide a center at the origin.

Proof. System (1) with $d=9, \lambda=B=C=0$ and $\operatorname{Re}\left(A^{4} D\right) \neq 0$ becomes

$$
\dot{z}=i z+z \bar{z}\left(A z^{5} \bar{z}^{2}+D \bar{z}^{7}\right) .
$$

Now if we make the change $z \rightarrow w=\xi z$ with $\xi=\bar{A}^{3 / 16} / A^{5 / 16}$ and use (6), then we have that system (12) can be written as

$$
\dot{w}=i w+w \bar{w}\left(w^{5} \bar{w}^{2}+\tilde{D} \bar{w}^{7}\right), \quad \tilde{D}=\frac{D A^{3 / 2}}{\bar{A}^{5 / 2}} \in \mathbb{C},
$$

with the condition $\operatorname{Re}(\tilde{D}) \neq 0$. We write $\tilde{D}=\tilde{d}_{1}+i \tilde{d}_{2}$. For system (13) (in view of Proposition 3) we have that $V_{2}=\cdots=V_{7}=0$. Now using $\rho_{1}, \ldots, \rho_{7}$ computed in the proof of Proposition 3 and using that

$$
\begin{aligned}
\rho_{8}^{\prime}= & 3 A\left(\rho_{2} \rho_{3}^{2}+\rho_{2}^{2} \rho_{4}+2 \rho_{1} \rho_{3} \rho_{4}+2 \rho_{1} \rho_{2} \rho_{5}+\rho_{1}^{2} \rho_{6}\right) \\
& +B\left(\rho_{4}^{2}+2 \rho_{3} \rho_{5}+2 \rho_{2} \rho_{6}+2 \rho_{1} \rho_{7}\right),
\end{aligned}
$$

we get that

$$
V_{8}=\tilde{d}_{1}\left(295|\tilde{D}|^{2}-252\right),
$$

which $V_{8} \equiv \rho_{8}(2 \pi)$ (mod. $\left.\left\{V_{1}, V_{2}, \ldots, V_{7}\right\}\right)$, and also modulo a positive constant. Therefore, in order that $V_{8}=0$, since $\tilde{d}_{1} \neq 0$, we need to impose that

$$
|\tilde{D}|^{2}=\frac{252}{295}, \quad \text { that is } \quad \tilde{d}_{1}=\sqrt{\frac{252}{295}} \cos (\psi), \quad \tilde{d}_{2}=\sqrt{\frac{252}{295}} \sin (\psi),
$$

with $\psi \in[0,2 \pi) \backslash\{\pi / 2,3 \pi / 2\}$. Therefore condition (c.3) becomes

$$
\text { (c.3)' } \lambda=B=C=0, \tilde{D}=\sqrt{\frac{252}{295}} e^{i \psi}, \psi \in[0,2 \pi) \backslash\{\pi / 2,3 \pi / 2\} .
$$

Now (13) becomes

$$
\dot{w}=i w+w \bar{w}\left(w^{5} \bar{w}^{2}+\sqrt{\frac{252}{295}} e^{i \psi} \bar{w}^{7}\right), \quad \psi \in[0,2 \pi) \backslash\{\pi / 2,3 \pi / 2\} .
$$

For this system and using that

$$
\begin{aligned}
\rho_{9}^{\prime}= & A\left(\rho_{3}^{3}+6 \rho_{2} \rho_{3} \rho_{4}+3 \rho_{1} \rho_{4}^{2}+3 \rho_{2}^{2} \rho_{5}+6 \rho_{1} \rho_{3} \rho_{5}+6 \rho_{1} \rho_{2} \rho_{6}+3 \rho_{1}^{2} \rho_{7}\right) \\
& +2 B\left(\rho_{4} \rho_{5}+\rho_{3} \rho_{6}+\rho_{2} \rho_{7}+\rho_{1} \rho_{8}\right), \\
\rho_{10}^{\prime}= & 3 A\left(\rho_{3}^{2} \rho_{4}+\rho_{2} \rho_{4}^{2}+2 \rho_{2} \rho_{3} \rho_{5}+2 \rho_{1} \rho_{4} \rho_{5}+\rho_{2}^{2} \rho_{6}+2 \rho_{1} \rho_{3} \rho_{6}+2 \rho_{1} \rho_{2} \rho_{7}\right. \\
& \left.+\rho_{1}^{2} \rho_{8}\right)+B\left(\rho_{5}^{2}+2 \rho_{4} \rho_{6}+2 \rho_{3} \rho_{7}+2 \rho_{2} \rho_{8}+2 \rho_{1} \rho_{9}\right),
\end{aligned}
$$

we get that $V_{9}=0$ and $V_{10}=\cos (\psi)$, where $V_{k} \equiv \rho_{k}(2 \pi)\left(\bmod \left\{\left\{V_{1}, V_{2}, \ldots, V_{k-1}\right\}\right)\right.$ for $k=9,10$, and also modulo a positive constant. However by hypothesis we have that $\cos (\psi) \neq 0$ and thus $V_{10} \neq 0$. This implies that system (12) 
does not have a center at the origin and consequently condition (c.3) does not provide a center.

Proposition 6. Let $j=0$. Condition either (c.4), or (c.5), or (c.6) does not provide a center at the origin.

Proof. System (1) with $\lambda=B=0, C=\bar{A} / \mu$ (where $\mu$ is defined in the Case 2 of the proof of Proposition 4$)$ and $\operatorname{Re}\left(A^{4} D\right) \neq 0$ becomes

$$
\dot{z}=i z+(z \bar{z})^{\frac{d-7}{2}}\left(A z^{5} \bar{z}^{2}+\frac{1}{\mu} \bar{A} z^{3} \bar{z}^{4}+D \bar{z}^{7}\right) .
$$

Now if we make the change $z \rightarrow w=\xi z$ with $\xi=\bar{A}^{(d-3) /(4(d-1))} / A^{(d+1) /(4(d-1))}$ and use (6), then we have that system (16) can be written as

$$
\dot{w}=i w+(w \bar{w})^{\frac{d-7}{2}}\left(w^{5} \bar{w}^{2}+\frac{1}{\mu} w^{3} \bar{w}^{4}+\tilde{D} \bar{w}^{7}\right), \quad \tilde{D}=\frac{D A^{3 / 2}}{\bar{A}^{5 / 2}} \in \mathbb{C},
$$

with the condition $\operatorname{Re}(\tilde{D}) \neq 0$. We write $\tilde{D}=\tilde{d}_{1}+i \tilde{d}_{2}$. For system (17) (in view of Proposition 3 ) we have that $V_{2}=\cdots=V_{7}=0$. Now using $\rho_{1}, \ldots, \rho_{7}$ computed in Proposition 3 and using that $\rho_{8}$ satisfies (14) we get that

$$
V_{8}=\tilde{d}_{1}\left(R_{d}^{1}+R_{d}^{2}|\tilde{D}|^{2}\right)
$$

with $R_{d}^{1}$ and $R_{d}^{2}$ equal to

$$
R_{d}^{1}= \begin{cases}16128(d-1)^{2} & \text { if }(\text { c. } 4) \text { holds } \\ 6048(d-1)^{2} & \text { if }(\text { c.5 }) \text { holds } \\ 16896(d-1)^{2} & \text { if }(\text { c. } 6) \text { holds }\end{cases}
$$

and

$$
R_{d}^{2}= \begin{cases}5 d^{4}-190 d^{3}-6180 d^{2}-51730 d-136465 & \text { if (c.4) holds } \\ 55 d^{4}+110 d^{3}-4305 d^{2}-16780 d-16340 & \text { if (c.5) holds } \\ -1215 d^{4}-6030 d^{3}+7140 d^{2}+6590 d+1195 & \text { if (c.6) holds }\end{cases}
$$

We want to make $V_{8}=0$. Since $\tilde{d}_{1} \neq 0$ we get that $|\tilde{D}|^{2}=-R_{d}^{1} / R_{d}^{2}$. Since $R_{d}^{1}>0$ we have to restrict to the values of $d$ for which $R_{d}^{2}<0$. Therefore, in order that $V_{8}=0$ we need to impose that

$$
\tilde{D}=\sqrt{\frac{R_{d}^{1}}{-R_{d}^{2}}} e^{i \psi}, \quad \psi \in[0,2 \pi) \backslash\{\pi / 2,3 \pi / 2\},
$$

with some restrictions on $d$ such that $R_{d}^{2}<0$. Therefore conditions (c.4), (c.5) and (c.6) become

$$
\begin{aligned}
& \text { (c.4)' } \lambda=B=(d-9) A+(d+7) \bar{C}=0, \tilde{D}=\sqrt{R_{d}^{1} /\left(-R_{d}^{2}\right)} e^{i \psi}, \psi \in \\
& {[0,2 \pi) \backslash\{\pi / 2,3 \pi / 2\} \text { and } d \in\{7,9, \ldots, 61\} ;} \\
& \left(\text { c.5)' } \lambda=B=(d-4) A+(d+2) \bar{C}=0, \tilde{D}=\sqrt{R_{d}^{1} /\left(-R_{d}^{2}\right)} e^{i \psi}, \psi \in\right. \\
& \\
& {[0,2 \pi) \backslash\{\pi / 2,3 \pi / 2\} \text { and } d \in\{7,9\} ;}
\end{aligned}
$$




$$
\begin{aligned}
\text { (c.6) } & \lambda=B=(3 d-7) A+(3 d+1) \bar{C}=0, \tilde{D}=\sqrt{R_{d}^{1} /\left(-R_{d}^{2}\right)} e^{i \psi}, \psi \in \\
& {[0,2 \pi) \backslash\{\pi / 2,3 \pi / 2\} \text { and } d \geq 7 \text { odd; } }
\end{aligned}
$$

respectively. Now (17) becomes

$$
\dot{w}=i w+(w \bar{w})^{\frac{d-7}{2}}\left(w^{5} \bar{w}^{2}+\frac{1}{\mu} w^{3} \bar{w}^{4}+\sqrt{\frac{R_{d}^{1}}{-R_{d}^{2}}} e^{i \psi} \bar{w}^{7}\right),
$$

with $\psi \in[0,2 \pi) \backslash\{\pi / 2,3 \pi / 2\}$ and the corresponding restrictions on the values of $d$ given above. From (18) using the equations for $\rho_{9}$ and $\rho_{10}$ in (15) we get that $V_{9}=0$ and $V_{10}=\cos (\psi)$. However by hypothesis we have that $\cos (\psi) \neq 0$ and thus $V_{10} \neq 0$. This implies that system (16) does not have a center at the origin and consequently conditions either (c.4), or (c.5), or (c.6) does not provide a center.

Proposition 7. Let $j=1$. The Liapunov constants of system (1), with $d \geq 9$ odd, are

$$
\begin{aligned}
V_{1}= & e^{2 \pi(d-1) \lambda} \\
V_{2}= & b_{1}, \\
V_{3}= & -\operatorname{Im}(A C), \\
V_{4}=0 & \\
V_{5}= & 0, \\
V_{6}= & 0, \\
V_{7}= & -\operatorname{Im}((3 A+2 \bar{C}) D[(d-2) A+d \bar{C}][(d-11) A+(d+9) \bar{C}] \\
& \quad[(d-5) A+(d+3) \bar{C}][(d-3) A+(d+1) \bar{C}]) \\
V_{8}= & \operatorname{Re}\left(( 3 A + 2 \overline { C } ) B D [ A - \overline { C } ] \left[\left(25 d^{3}-284 d^{2}+974 d-1023\right) A^{3}\right.\right. \\
& \quad+\left(75 d^{3}-434 d^{2}+162 d+1121\right) A^{2} \bar{C} \\
& \quad+\left(75 d^{3}-16 d^{2}-674 d-309\right) A \bar{C} \\
& \left.\left.\quad+\left(25 d^{3}+134 d^{2}+138 d+11\right) \bar{C}^{3}\right]\right),
\end{aligned}
$$

We remark that $V_{k} \equiv \rho_{k}(2 \pi)\left(\bmod .\left\{V_{1}, V_{2}, \ldots, V_{k-1}\right\}\right)$, for $k=1, \ldots, 8$ and also modulo a positive constant.

Proof. Proceeding in the same way as in the proof of Proposition 3 we readily get $V_{1}, V_{2}$ and $V_{3}$ in the statement in the proposition.

Computing the solution $\rho_{k}(\theta)$ from the differential equation for $\rho_{k}(\theta)$, we get $\rho_{k}(\theta)$ and in particular we obtain that $V_{k}=0$, being $V_{k}$ equal to $\rho_{k}(2 \pi)$ when $\rho_{2}(2 \pi)=\rho_{k-1}(2 \pi)=0$ for $k=4,5,6$.

Solving the differential equation for $\rho_{7}(\theta)$ we get $\rho_{7}(\theta)$ and in particular we obtain from the expression of $v_{7}=\rho_{7}(2 \pi)$ the value of $V_{7}$ given in the statement of Proposition 7 modulo $\rho_{2}(2 \pi)=\cdots=\rho_{6}(2 \pi)=0$ and a positive constant. More precisely we can check that if we multiply $v_{7}$ by $640 /((d-$ 
1) $\pi)$ then

$$
\begin{aligned}
& v_{7}=V_{7}-V_{3}\left(-56 a_{2} d_{2} a_{1}^{2} d^{4}+26 c_{2} d_{2} a_{1}^{2} d^{4}-216 a_{2} d_{2} c_{1}^{2} d^{4}+66 c_{2} d_{2} c_{1}^{2} d^{4}+\right. \\
& 56 a_{2}^{3} d_{2} d^{4}-11 c_{2}^{3} d_{2} d^{4}+48 a_{2} c_{2}^{2} d_{2} d^{4}-78 a_{2}^{2} c_{2} d_{2} d^{4}-182 a_{2} d_{2} a_{1} c_{1} d^{4}+ \\
& 72 c_{2} d_{2} a_{1} c_{1} d^{4}+14 a_{1}^{3} d_{1} d^{4}+44 c_{1}^{3} d_{1} d^{4}+72 a_{1} c_{1}^{2} d_{1} d^{4}-84 a_{2}^{2} a_{1} d_{1} d^{4}- \\
& 24 c_{2}^{2} a_{1} d_{1} d^{4}+78 a_{2} c_{2} a_{1} d_{1} d^{4}-234 a_{2}^{2} c_{1} d_{1} d^{4}-44 c_{2}^{2} c_{1} d_{1} d^{4}+52 a_{1}^{2} c_{1} d_{1} d^{4}+ \\
& 168 a_{2} c_{2} c_{1} d_{1} d^{4}+768 a_{2} d_{2} a_{1}^{2} d^{3}-172 c_{2} d_{2} a_{1}^{2} d^{3}-54 a_{2} d_{2} c_{1}^{2} d^{3}+ \\
& 450 c_{2} d_{2} c_{1}^{2} d^{3}-768 a_{2}^{3} d_{2} d^{3}-75 c_{2}^{3} d_{2} d^{3}+12 a_{2} c_{2}^{2} d_{2} d^{3}+516 a_{2}^{2} c_{2} d_{2} d^{3}+ \\
& 1204 a_{2} d_{2} a_{1} c_{1} d^{3}+18 c_{2} d_{2} a_{1} c_{1} d^{3}-192 a_{1}^{3} d_{1} d^{3}+300 c_{1}^{3} d_{1} d^{3}+ \\
& 18 a_{1} c_{1}^{2} d_{1} d^{3}+1152 a_{2}^{2} a_{1} d_{1} d^{3}-6 c_{2}^{2} a_{1} d_{1} d^{3}-516 a_{2} c_{2} a_{1} d_{1} d^{3}+ \\
& 1548 a_{2}^{2} c_{1} d_{1} d^{3}-300 c_{2}^{2} c_{1} d_{1} d^{3}-344 a_{1}^{2} c_{1} d_{1} d^{3}+42 a_{2} c_{2} c_{1} d_{1} d^{3}- \\
& 2640 a_{2} d_{2} a_{1}^{2} d^{2}-144 c_{2} d_{2} a_{1}^{2} d^{2}+4482 a_{2} d_{2} c_{1}^{2} d^{2}-234 c_{2} d_{2} c_{1}^{2} d^{2}+ \\
& 2640 a_{2}^{3} d_{2} d^{2}+39 c_{2}^{3} d_{2} d^{2}-996 a_{2} c_{2}^{2} d_{2} d^{2}+432 a_{2}^{2} c_{2} d_{2} d^{2}+ \\
& 1008 a_{2} d_{2} a_{1} c_{1} d^{2}-1494 c_{2} d_{2} a_{1} c_{1} d^{2}+660 a_{1}^{3} d_{1} d^{2}-156 c_{1}^{3} d_{1} d^{2}- \\
& 1494 a_{1} c_{1}^{2} d_{1} d^{2}-3960 a_{2}^{2} a_{1} d_{1} d^{2}+498 c_{2}^{2} a_{1} d_{1} d^{2}-432 a_{2} c_{2} a_{1} d_{1} d^{2}+ \\
& 1296 a_{2}^{2} c_{1} d_{1} d^{2}+156 c_{2}^{2} c_{1} d_{1} d^{2}-288 a_{1}^{2} c_{1} d_{1} d^{2}-3486 a_{2} c_{2} c_{1} d_{1} d^{2}+ \\
& 832 a_{2} d_{2} a_{1}^{2} d+1292 c_{2} d_{2} a_{1}^{2} d+54 a_{2} d_{2} c_{1}^{2} d-2034 c_{2} d_{2} c_{1}^{2} d-832 a_{2}^{3} d_{2} d+ \\
& 339 c_{2}^{3} d_{2} d-12 a_{2} c_{2}^{2} d_{2} d-3876 a_{2}^{2} c_{2} d_{2} d-9044 a_{2} d_{2} a_{1} c_{1} d- \\
& 18 c_{2} d_{2} a_{1} c_{1} d-208 a_{1}^{3} d_{1} d-1356 c_{1}^{3} d_{1} d-18 a_{1} c_{1}^{2} d_{1} d+1248 a_{2}^{2} a_{1} d_{1} d+ \\
& 6 c_{2}^{2} a_{1} d_{1} d+3876 a_{2} c_{2} a_{1} d_{1} d-11628 a_{2}^{2} c_{1} d_{1} d+1356 c_{2}^{2} c_{1} d_{1} d+ \\
& 2584 a_{1}^{2} c_{1} d_{1} d-42 a_{2} c_{2} c_{1} d_{1} d+4296 a_{2} d_{2} a_{1}^{2}-202 c_{2} d_{2} a_{1}^{2}-4266 a_{2} d_{2} c_{1}^{2}- \\
& 648 c_{2} d_{2} c_{1}^{2}-4296 a_{2}^{3} d_{2}+108 c_{2}^{3} d_{2}+948 a_{2} c_{2}^{2} d_{2}+606 a_{2}^{2} c_{2} d_{2}+ \\
& 1414 a_{2} d_{2} a_{1} c_{1}+1422 c_{2} d_{2} a_{1} c_{1}-1074 a_{1}^{3} d_{1}-432 c_{1}^{3} d_{1}+1422 a_{1} c_{1}^{2} d_{1}+ \\
& 6444 a_{2}^{2} a_{1} d_{1}-474 c_{2}^{2} a_{1} d_{1}-606 a_{2} c_{2} a_{1} d_{1}+1818 a_{2}^{2} c_{1} d_{1}+432 c_{2}^{2} c_{1} d_{1}- \\
& \left.404 a_{1}^{2} c_{1} d_{1}+3318 a_{2} c_{2} c_{1} d_{1}\right) \text {. }
\end{aligned}
$$

We compute the solution $\rho_{8}(\theta)$ from the differential equation for $\rho_{8}(\theta)$ (see (14)), we get $\rho_{8}(\theta)$, and in particular we obtain the expression for $v_{8}=$ $\rho_{8}(2 \pi)$ given in the statement of Proposition 7 modulo $\rho_{2}(2 \pi)=\rho_{3}(2 \pi)=$ $\rho_{6}(2 \pi)=\rho_{7}(2 \pi)=0$ and a positive constant. The computation of $V_{8}$ is done in the same way as $V_{7}$. This completes the proof of the proposition.

Proposition 8. Let $j=1$. For $d \geq 9$ odd if $V_{1}=1$, and $V_{k}=0$ for $f=2, \ldots, 8$, then one of the following conditions holds.

(a.1) $\lambda=b_{1}=3 A+2 \bar{C}=0$,

(a.3) $\lambda=b_{1}=\operatorname{Im}(A C)=\operatorname{Im}\left(A^{5} D\right)=\operatorname{Im}\left(\bar{C}^{5} D\right)=0$, 
(d.3) $\lambda=B=C=0, d=11$ and $\operatorname{Im}\left(A^{5} D\right) \neq 0$,

(d.4) $\lambda=B=(d-11) A+(d+9) \bar{C}=0, d \neq 11$ and condition (a.3) does not hold, does not hold,

(d.5) $\lambda=B=(d-2) A+d \bar{C}=0$ and condition (a.3) does not hold,

(d.6) $\lambda=B=(d-3) A+(d+1) \bar{C}=0$ and condition (a.3) does not hold,

(d.7) $\lambda=B=(d-5) A+(d+3) \bar{C}=0$ and condition (a.3) does not hold,

Proof. From the fact that $V_{1}=1$ we get that $\lambda=0$. The condition $V_{2}=0$ implies that $b_{1}=0$. Furthermore to make $V_{3}=0$ we will consider two different cases: $C=0$ and $C \neq 0$. In this last case we have that $A=\mu \bar{C}$, with $\mu \in \mathbb{R}$.

Case 1: $C=0$. Then

$$
V_{7}=3(d-2)(d-11)(d-5)(d-3) \operatorname{Im}\left(A^{5} D\right) .
$$

In view of the factors of $V_{7}$ and since $d \geq 9$ odd, we need to consider two different subcases.

Subcase 1.1: $\operatorname{Im}\left(A^{5} D\right)=0$. Therefore we are under the hypotheses of condition (a.3).

Subcase $1.2: \operatorname{Im}\left(A^{5} D\right) \neq 0$ and $d=11$. In this case, we have

$$
V_{8}=25806 b_{2} \operatorname{Im}\left(A^{5} D\right) \text {. }
$$

To have $V_{8}=0$ we must impose $b_{2}=0$, that is, $B=0$. In this case we are under the hypotheses of condition (d.3).

Case 2: $A=\mu \bar{C}, \mu \in \mathbb{R}$. So

$$
\begin{aligned}
V_{7}= & (3 \mu+2) \operatorname{Im}\left(\bar{C}^{5} D\right)[((d-2) \mu+d)((d-11) \mu+d+9)((d-5) \mu+d+3)] \\
& {[(d-3) \mu+d+1] . }
\end{aligned}
$$

In view of the factors in $V_{7}$ we need to consider six different subcases.

Subcase 2.1: $\mu=-2 / 3$. In this case we are under the hypotheses of condition (a.1).

Subcase 2.2: $\operatorname{Im}\left(\bar{C}^{5} D\right)=0$. We are under the hypotheses of condition (a.3).

Subcase 2.3: $\mu=-d /(d-2)$ and $\operatorname{Im}\left(\bar{C}^{5} D\right) \neq 0$. Since $b_{1}=0$, we have

$$
V_{8}=\frac{176(d-1)^{4}(d+4)}{(d-2)^{5}} b_{2} \operatorname{Im}\left(\bar{C}^{5} D\right) \text {. }
$$

Then, since $d \geq 11$ odd, we get that $V_{8}=0$ if and only if $b_{2}=0$, that is $B=0$. Hence we are under the hypothesis of condition (d.5).

Subcase 2.4: $\mu=-(d+9) /(d-11), \operatorname{Im}\left(\bar{C}^{5} D\right) \neq 0$ and $d \neq 11$. Therefore since $b_{1}=0$, we have

$$
V_{8}=-\frac{137632(d-1)^{4}(d+49)}{(d-11)^{5}} b_{2} \operatorname{Im}\left(\bar{C}^{5} D\right) .
$$


Then, since $d \geq 9$ odd, $d \neq 11$, we get that $V_{8}=0$ if and only if $b_{2}=0$, that is $B=0$. Then we are under the hypothesis of condition (d.4).

Subcase 2.5: $\mu=-(d+3) /(d-5), \operatorname{Im}\left(\bar{C}^{5} D\right) \neq 0$. Since $b_{1}=0$, we have

$$
V_{8}=\frac{2048(d-1)^{4}(d+19)}{(d-5)^{5}} b_{2} \operatorname{Im}\left(\bar{C}^{5} D\right)
$$

Then, since $d \geq 9$ odd, we get that $V_{8}=0$ if and only if $b_{2}=0$, that is $B=0$. In this case we are under the hypothesis of condition (d.7).

Subcase 2.6: $\mu=-(d+1) /(d-3), \operatorname{Im}\left(\bar{C}^{5} D\right) \neq 0$. Since $b_{1}=0$, we have

$$
V_{8}=-\frac{288(d-1)^{4}(d+9)}{(d-3)^{5}} b_{2} \operatorname{Im}\left(\bar{C}^{5} D\right) .
$$

Then, since $d \geq 9$ odd, we get that $V_{8}=0$ if and only if $b_{2}=0$, that is $B=0$. In this case we are under the hypothesis of condition (d.6).

Now we show that conditions (d.k) with $k=3, \ldots, 7$ do not provide a center at the origin.

Proposition 9. Let $j=1$. Condition (d.3) does not provide a center at the origin.

Proof. System (1) with $d=11, \lambda=B=C=0$ and $\operatorname{Im}\left(A^{5} D\right) \neq 0$ becomes

$$
\dot{z}=i z+z \bar{z}\left(A z^{6} \bar{z}^{3}+D \bar{z}^{9}\right) .
$$

Now if we make the change $z \rightarrow w=\xi z$ with $\xi=\bar{A}^{1 / 5} / A^{3 / 10}$ and using (6) then we have that system (19) can be written as

$$
\dot{w}=i w+w \bar{w}\left(w^{6} \bar{w}^{3}+\tilde{D} \bar{w}^{9}\right), \quad \tilde{D}=\frac{D A^{2}}{\bar{A}^{3}} \in \mathbb{C},
$$

with the condition $\operatorname{Im}(\tilde{D}) \neq 0$. For system (20) (in view of Proposition 7) we have that $V_{1}=\cdots=V_{8}=0$. Now using $\rho_{1}, \ldots, \rho_{8}$ computed in the proof of Proposition 7 and using (15) we get that

$$
V_{9}=\tilde{d}_{2}\left(9|\tilde{D}|^{2}-8\right)
$$

which $V_{9} \equiv \rho_{9}(2 \pi)\left(\bmod .\left\{V_{2}, \ldots, V_{8}\right\}\right)$, and also modulo a positive constant. Therefore, in order that $V_{9}=0$ we also need to impose that

$$
\tilde{D}=\frac{2 \sqrt{2}}{3} e^{i \psi}, \quad \psi \in(0,2 \pi) \backslash\{\pi\} .
$$

So condition (d.3) becomes

(d.3)' $\lambda=B=C=0, \tilde{D}=\frac{2 \sqrt{2}}{3} e^{i \psi}$ with $\psi \in(0,2 \pi) \backslash\{\pi\}$ and $d=11$.

Now (20) becomes

$$
\dot{w}=i w+w \bar{w}\left(w^{6} \bar{w}^{3}+\frac{2 \sqrt{2}}{3} e^{i \psi} \bar{w}^{9}\right), \quad \psi \in(0,2 \pi) \backslash\{\pi\} .
$$


For this system and using that $\rho_{10}$ was computed in (15) and using also

$$
\begin{aligned}
\rho_{11}^{\prime}= & 3 A\left(\rho_{3} \rho_{4}^{2}+\rho_{3}^{2} \rho_{5}+2 \rho_{2} \rho_{4} \rho_{5}+\rho_{1} \rho_{5}^{2}+2 \rho_{2} \rho_{3} \rho_{6}+2 \rho_{1} \rho_{4} \rho_{6}+\rho_{2}^{2} \rho_{7}\right. \\
& \left.+2 \rho_{1} \rho_{3} \rho_{7}+2 \rho_{1} \rho_{2} \rho_{8}+\rho_{1}^{2} \rho_{9}\right)+2 B\left(\rho_{1} \rho_{10}+\rho_{5} \rho_{6}+\rho_{4} \rho_{7}+\rho_{3} \rho_{8}+\rho_{2} \rho_{9}\right),
\end{aligned}
$$

we get that $V_{10}=0$ and $V_{11}=\sin (\psi)$, with $V_{k} \equiv \rho_{k}(2 \pi)\left(\bmod .\left\{V_{2}, \ldots, V_{k-1}\right\}\right)$ for $k=10,11$, and also modulo a positive constant. However by hypothesis we have that $\sin (\psi) \neq 0$ and thus $V_{11} \neq 0$. Consequently condition (d.3) does not provide a center.

Proposition 10. Let $j=1$. Condition either (d.4), or (d.5), or (d.6), or (d.7) does not provide a center at the origin.

Proof. System (1) with $\lambda=B=0, A=\mu \bar{C}$ (where $\mu$ is defined in the case 2 in the proof of Proposition 8 and $\operatorname{Im}\left(A^{5} D\right) \neq 0$ becomes

$$
\dot{z}=i z+(z \bar{z})^{\frac{d-9}{2}}\left(\mu \bar{C} z^{6} \bar{z}^{3}+C z^{4} \bar{z}^{5}+D \bar{z}^{9}\right) .
$$

Now if we make the change $z \rightarrow w=\xi z$ with $\xi=C^{(d-3) /(4(d-1))} / \bar{C}^{(d+1) /(4(d-1))}$ and use (6) then we have that system (22) can be written as

$$
\dot{w}=i w+(w \bar{w})^{\frac{d-9}{2}}\left(\mu w^{6} \bar{w}^{3}+w^{4} \bar{w}^{5}+\tilde{D} \bar{w}^{9}\right), \quad \tilde{D}=\frac{D \bar{C}^{2}}{C^{3}} \in \mathbb{C},
$$

with the condition $\operatorname{Im}(\tilde{D}) \neq 0$. For system (23) (in view of Proposition 7) we have that $V_{2}=\cdots=V_{8}=0$. Now using $\rho_{1}, \ldots, \rho_{8}$ computed in the proof of Proposition 3 and using that $\rho_{9}$ satisfies (14) we get that

$$
V_{9}=\tilde{d}_{2}\left(R_{d}^{1}+R_{d}^{2}|\tilde{D}|^{2}\right),
$$

with $R_{d}^{1}$ and $R_{d}^{2}$ equal to

$$
R_{d}^{1}= \begin{cases}7680(d-1)^{2} & \text { if we are in condition (d.4) } \\ -420(d-1)^{2} & \text { if we are in condition (d.5) } \\ 0 & \text { if we are in condition (d.6) } \\ 33600(d-1)^{2} & \text { if we are in condition (d.7) }\end{cases}
$$

and

$$
R_{d}^{2}= \begin{cases}d^{4}-116 d^{3}+942 d^{2}+16060 d-150887 & \text { if we are in condition (d.4), } \\ 28 d^{4}+46 d^{3}-651 d^{2}+456 d-524 & \text { if we are in condition (d.5), } \\ 1 & \text { if we are in condition (d.6) } \\ 73 d^{4}-1412 d^{3}-5466 d^{2}+124060 d-352775 & \text { if we are in condition (d.7) }\end{cases}
$$

We want to make $V_{9}=0$. Then since $\tilde{d}_{2} \neq 0$, we have that $V_{9} \neq 0$ if condition (d.6) is satisfied. Therefore condition (d.6) does not provide a center. 
For conditions (d.4), (d.5) and (d.7) we have that $V_{9}=0$ implies $|\tilde{D}|^{2}=$ $-R_{d}^{1} / R_{d}^{2}$. Since $R_{d}^{1}>0$ in conditions (d.4) and (d.7) while $R_{d}^{1}<0$ in condition (d.5), we must restrict the values of $d$ for which $R_{d}^{2}<0$ in conditions (d.4) and (d.7) and $R_{d}^{2}>0$ in condition (d.5). Therefore

$$
\tilde{D}=\sqrt{\frac{R_{d}^{1}}{-R_{d}^{2}}} e^{i \psi}, \quad \psi \in(0,2 \pi) \backslash\{\pi\},
$$

and with the restriction on $d$ explained above. So conditions (d.4), (d.5) and (d.7) become

(d.4)' $\lambda=B=0,(d-11) A+(d+9) \bar{C}=0, \tilde{D}=\sqrt{R_{d}^{1} /\left(-R_{d}^{2}\right)} e^{i \psi}, \psi \in$ $(0,2 \pi) \backslash\{\pi\}$ and $d \in\{11,13, \ldots, 105\} ;$

(d.5)' $\lambda=B=0,(d-2) A+d \bar{C}=0, \tilde{D}=\sqrt{R_{d}^{1} /\left(-R_{d}^{2}\right)} e^{i \psi}, \psi \in(0,2 \pi) \backslash\{\pi\}$ and $d \geq 9$ odd;

(d.7)' $\lambda=B=0,(d-5) A+(d+3) \bar{C}=0, \tilde{D}=\sqrt{R_{d}^{1} /\left(-R_{d}^{2}\right)} e^{i \psi}, \psi \in$ $(0,2 \pi) \backslash\{\pi\}$ and $d \in\{7,9, \ldots, 19\} ;$

respectively. Now (23) becomes

$$
\dot{w}=i w+(w \bar{w})^{\frac{d-9}{2}}\left(\mu w^{6} \bar{w}^{3}+w^{4} \bar{w}^{5}+\sqrt{\frac{R_{d}^{1}}{-R_{d}^{2}}} e^{i \psi} \bar{w}^{9}\right),
$$

with $\psi \in(0,2 \pi) \backslash\{\pi\}$ and the corresponding restrictions on the parameter $d$ given above. From (24) using the equations for $\rho_{10}$ and $\rho_{11}$ of (15) and (21), we get that $V_{10}=0$ and $V_{11}=\sin (\psi)$. However by hypothesis we have that $\sin (\psi) \neq 0$ and thus $V_{11} \neq 0$. This implies that system (24) does not have a center at the origin and consequently condition either (d.4), or (d.5), or (d.7) does not provide a center.

\section{Proof of Theorem 1(B)}

We divide the proof of Theorem 1(b) in two different parts.

4.1. Sufficient conditions for an isochronous center. In this subsection we will see that conditions (b.1) and (b.2) are sufficient to have an isochronous center. For this we will prove that under conditions (b.1), or (b.2) equation (7) holds.

Since in the assumptions (b.1), or (b.2), we can assume that $A \neq 0$ (otherwise we will obtain a linear center), we can make the change of variables

$$
\omega=\xi z \quad \text { where } \quad \xi=\left(\frac{\bar{A}^{d-3}}{A^{d+1}}\right)^{1 /(4(d-1)},
$$

and system (1) with hypothesis (b.1) becomes

$$
w^{\prime}=i w+(w \bar{w})^{(d-7-2 j) / 2}\left(w^{5+j} \bar{w}^{2+j}+w^{3+j} \bar{w}^{4+j}\right) .
$$


Rewriting (26) in polar coordinates we obtain

$$
r^{\prime}=2 r^{d} \cos (2 \theta) \text { and } \quad \theta^{\prime}=1 .
$$

and clearly (7) holds.

Now system (1) with hypothesis (b.2) becomes

$$
w^{\prime}=i w+(w \bar{w})^{(d-7-2 j) / 2}\left(w^{5+j} \bar{w}^{2+j}+\frac{3-d}{d+1} w^{3+j} \bar{w}^{4+j}\right) .
$$

In polar coordinates it has the form

$$
r^{\prime}=\frac{4}{d+1} r^{d} \cos (2 \theta) \quad \text { and } \quad \theta^{\prime}=1+\frac{2(d-1)}{d+1} r^{d-1} \sin (2 \theta) .
$$

Therefore

$$
\frac{d r}{d \theta}=\frac{4 r^{d} \cos (2 \theta)}{d+1+2(d-1) r^{d-1} \sin (2 \theta)} \quad \text { with } \quad r(0)=r_{0}
$$

Then integrating it and since $r(\theta) \geq 0$ for any $\theta$ we get that

$$
r(\theta)=\left(\frac{-2(d-1) \sin (2 \theta)+\sqrt{(d+1)^{2} r_{0}^{2-2 d}+4(d-1)^{2} \sin ^{2}(2 \theta)}}{d+1}\right)^{1 /(1-d)}
$$

Note that

$$
\sqrt{(d+1)^{2} r_{0}^{2-2 d}+4(d-1)^{2} \sin ^{2}(2 \theta)} \geq|2(d-1) \sin (2 \theta)|
$$

and thus $r(\theta)$ given in (28) is positive. Therefore, introducing (28) into (27) we have that

$$
\int_{0}^{2 \pi} \frac{d \theta}{\theta^{\prime}}=\int_{0}^{2 \pi}\left(1-\frac{2(d-1) \sin (2 \theta)}{\sqrt{(d+1)^{2} r_{0}^{2-2 d}+4(d-1)^{2} \sin ^{2}(2 \theta)}}\right) d \theta=2 \pi
$$

since the function $2(d-1) \sin (2 \theta) / \sqrt{(d+1)^{2} r_{0}^{2-2 d}+4(d-1)^{2} \sin ^{2}(2 \theta)}$ is odd in $\theta$.

4.2. Necessary conditions for an isochronous center. In this subsection we will see that conditions (b.1), or (b.2) are necessary to have an isochronous center. For this we will first compute the period constants up to some order and then show that the zeros of those period constants are precisely conditions (b.1), or (b.2).

We note that since $\rho_{1}(\theta)=1$, then from (3) and (8) we have $T_{1}=2 \pi b_{2}$. Since from the conditions to be a center we have that $b_{1}=0$ from now on we will assume that $B=0$.

Now we compute $T_{2}$, using $\rho_{2}(\theta)$ computed in the proof of Proposition 3 and equations (3) and (8), we get

$$
T_{2}=4(d-3)|A|^{2}+16 \operatorname{Re}(A C)-4(d+1)|C|^{2}-(d+7)|D|^{2},
$$

up to a non-zero constant. We distinguish two different cases. 
Case 1: $A=0$. Then $T_{2}$ becomes

$$
T_{2}=-\left(4(d+1)|C|^{2}+(d+7)|D|^{2}\right) .
$$

In order that $T_{2}=0$ we must impose $C=D=0$. Then $A=B=C=$ $D=0$, and the system (1) becomes linear. Therefore we do not consider this case.

Case 2: $A \neq 0$. Since from $V_{2}=0$ we have that $\operatorname{Im}(A C)=0$, we get that $C=\mu \bar{A}$ with $\mu \in \mathbb{R}$. We will consider two different subcases.

Subcase 2.1: $\mu=-(5+j) /(3+j)$. Therefore $C=-(5+j) \bar{A} /(3+j)$ and we are under the hypothesis (a.1). Then $T_{2}$ becomes

$$
T_{2}=-\frac{(d+7)}{9}\left(64|A|^{2}+9|D|^{2}\right) .
$$

Since $A \neq 0$, we get that $T_{2} \neq 0$. Therefore this case does not provide an isochronous center.

Subcase 2.2: $\mu \in \mathbb{R} \backslash\{-(5+j) /(3+j)\}$. Now $C=\mu \bar{A}$ and we are under the conditions either (a.2) or (a.3). We consider two different subcases.

Subcase 2.2.1: $j=0$. By the change of variables in (25) we can rewrite system (1) as

$$
w^{\prime}=i w+(w \bar{w})^{(d-7) / 2}\left[w^{5} \bar{w}^{2}+\mu w^{3} \bar{w}^{4}+\tilde{D} \bar{w}^{7}\right], \quad \tilde{D}=\frac{D A^{3 / 2}}{\bar{A}^{5 / 2}} .
$$

Since we are in assumptions (a.2) we have that $\operatorname{Re}\left(A^{4} D\right)=0$. Therefore

$$
\tilde{d}_{1}=\operatorname{Re}(\tilde{D})=\operatorname{Re}\left(\frac{D A^{3 / 2}}{\bar{A}^{5 / 2}}\right)=\frac{1}{|A|^{5}} \operatorname{Re}\left(A^{4} D\right)=0 .
$$

In what follows we denote $\tilde{d}_{2}$ simply by $d_{2}$. Computing $T_{k}$ for $k=2,3,4,5$ we get

$$
\begin{aligned}
T_{2}= & 4(d-3)+16 \mu-4(d+1) \mu^{2}-(d+7) d_{2}^{2}, \\
T_{3}= & 0, \\
T_{4}= & 225 d^{3} \mu^{4}+1425 d^{2} \mu^{4}-1089 d \mu^{4}-2289 \mu^{4}+360 d^{3} \mu^{3}-1952 d^{2} \mu^{3}- \\
& 8040 d \mu^{3}+7328 \mu^{3}-90 d^{3} \mu^{2}-3570 d^{2} \mu^{2}-4608 d_{2}^{2} \mu^{2}+19242 d \mu^{2}+ \\
& 9890 \mu^{2}-360 d^{3} \mu+1952 d^{2} \mu+4552 d \mu-43264 \mu-135 d^{3}+ \\
& 2145 d^{2}+12800 d_{2}^{2}-14665 d+28335, \\
T_{5}= & -d_{2}\left(-1260 \mu^{3} d^{4}-3060 \mu^{2} d^{4}-2340 \mu d^{4}-540 d^{4}-5678 \mu^{3} d^{3}+\right. \\
& 6450 \mu^{2} d^{3}+20078 \mu d^{3}+7950 d^{3}-3231 \mu^{4} d^{2}+1114 \mu^{3} d^{2}+ \\
& 43608 \mu^{2} d^{2}-36666 \mu d^{2}-48025 d^{2}+42390 \mu^{4} d-658 \mu^{3} d- \\
& 156222 \mu^{2} d-58830 \mu d+202120 d+42921 \mu^{4}-183598 \mu^{3}- \\
& \left.192000 d_{2}^{2}+69120 d_{2}^{2} \mu^{2}+64104 \mu^{2}+409918 \mu-340545\right),
\end{aligned}
$$

where the period constant $T_{k}$ has been computed modulo the constants $T_{l}=0$ for $l=2, \ldots, k-1$ and modulo a non-zero constant. 
The period constants $T_{2}, T_{4}$ and $T_{5}$ are polynomials in the variables $d, d_{2}$ and $\mu$. We want to study the zeros $\left(d, d_{2}, \mu\right)$ of $T_{2}, T_{4}$ and $T_{5}$ with $d \geq 7$ an odd positive integer. For doing that we consider the resultant of $T_{2}$ and $T_{4}$ with respect to $\mu$. This resultant is a polynomial $f_{1}$ in the variables $d$ and $d_{2}$. After we consider the resultant of $T_{2}$ and $T_{5}$ with respect to $\mu$. This resultant is a polynomial $f_{2}$ in the variables $d$ and $d_{2}$. The polynomials $f_{1}$ and $f_{2}$ have in common the factors $d_{2}$. We define the polynomials $g_{1}$ and $g_{2}$ as the polynomials $f_{1}$ and $f_{2}$ omitting the common factor $d_{2}$. Then we consider the resultant of $g_{1}$ and $g_{2}$ with respect to $d_{2}$. This resultant is a polynomial $h$ in the variable $d$. It easy to check that the unique positive odd integer root $\geq 7$ of the polynomial $h$ is $d=17$. In short the common zeros $\left(d, d_{1}, \mu\right)$ of $T_{2}, T_{4}$ and $T_{5}$ must have either $d_{2}=0$, or $d=17$.

Assume $d_{2}=0$. Then $T_{2}=4(1-\mu)(d-3+(d+1) \mu)$ and $T_{2}$ divides $T_{4}$ and $T_{5}$. So $d_{2}=0$ and either $\mu=1$ or $\mu=(3-d) /(d+1)$ vanish $T_{2}, T_{4}$ and $T_{5}$. The case $d_{2}=0$ and $\mu=1$ corresponds to the condition (b.1) of Theorem 1. The case $d_{2}=0$ and $\mu=(3-d) /(d+1)$ corresponds to the condition (b.2) of Theorem 1. Hence these two conditions are necessary for having an isochronous center.

Assume $d=17$ and $d_{2} \neq 0$. Then

$$
\begin{aligned}
T_{2}= & -8\left(3 d_{2}^{2}+9 \mu^{2}-2 \mu-7\right), \\
T_{4}= & -128\left(-11691 \mu^{4}-8400 \mu^{3}+36 d_{2}^{2} \mu^{2}+8882 \mu^{2}+\right. \\
& \left.9144 \mu-100 d_{2}^{2}+2065\right) .
\end{aligned}
$$

Doing the resultant of $T_{2}$ and $T_{4}$ with respect to $d_{1}$ we obtain the polynomial

$$
1048576(m-1)^{2}(3 m+1)^{2}(9 m+7)^{2}(1311 m+785)^{2} .
$$

Substituting $d=17$ and $\mu$ for every one of the four roots of the previous polynomial in $T_{2}, T_{4}$ and $T_{5}$ we get three polynomials in the variable $d_{2}$. Taking into account that $d_{2} \neq 0$, the unique set of the three polynomials which have a common root is the set corresponding to $d=17$ and $\mu=-1 / 3$. The common roots are $d_{2}= \pm 4 / 3$. But computing $T_{6}$ and evaluating it at $\left(d, \mu, d_{2}\right)=(17,-1 / 3, \pm 4 / 3)$, it is not zero. Consequently there are no more candidates for isochronous centers. This completes the proof of Theorem 1(b) when $j=0$.

Subcase 2.2.2: $j=1$. By the change of variables in (25) we can rewrite system (1) as

$$
w^{\prime}=i w+(w \bar{w})^{(d-9) / 2}\left[w^{6} \bar{w}^{3}+\mu w^{4} \bar{w}^{5}+\tilde{D} \bar{w}^{9}\right], \quad \tilde{D}=\frac{D A^{2}}{\bar{A}^{3}} .
$$

Since we are in the assumptions of condition (a.3) we have that $\operatorname{Im}\left(A^{5} D\right)=$ 0 . Therefore,

$$
\tilde{d}_{2}=\operatorname{Im}(\tilde{D})=\frac{1}{|A|^{6}} \operatorname{Im}\left(D A^{5}\right)=0
$$


In what follows we write $d_{2}$ instead of $\tilde{d}_{2}$. Computing $T_{k}$ for $k=2,3,4,5,6$ we get

$$
\begin{aligned}
& T_{2}=-d d_{1}^{2}-9 d_{1}^{2}-5 d \mu^{2}-5 \mu^{2}+5 d+20 \mu-15 \\
& T_{3}=0 \\
& T_{4}= 27 d^{3} \mu^{4}+177 d^{2} \mu^{4}-247 d \mu^{4}-397 \mu^{4}+45 d^{3} \mu^{3}-221 d^{2} \mu^{3}- \\
& 1005 d \mu^{3}+1341 \mu^{3}-9 d^{3} \mu^{2}-453 d^{2} \mu^{2}-800 d_{1}^{2} \mu^{2}+2541 d \mu^{2}+ \\
& 1081 \mu^{2}-45 d^{3} \mu+221 d^{2} \mu+661 d \mu-5877 \mu-18 d^{3}+276 d^{2}+ \\
& 1800 d_{1}^{2}-1950 d+3852, \\
& T_{5}= 0, \\
& T_{6}=-2374168320 d^{2} \mu^{6}+4737709440 d \mu^{6}+7111877760 \mu^{6}- \\
& 6700703760 d^{2} \mu^{5}+66277756320 d \mu^{5}+107348472 d^{2} d_{1} \mu^{5}- \\
& 466924920 d d_{1} \mu^{5}-574273392 d_{1} \mu^{5}+35034275760 \mu^{5}- \\
& 442707480 d^{2} \mu^{4}+14809132800 d_{1}^{2} \mu^{4}+88626216560 d \mu^{4}+ \\
& 542685528 d^{2} d_{1} \mu^{4}-4269915188 d d_{1} \mu^{4}-2086113260 d_{1} \mu^{4}- \\
& 153716853880 \mu^{4}+2592000 d^{4} \mu^{3}-19615500 d^{3} \mu^{3}- \\
& 1062415200 d_{1}^{3} \mu^{3}+10512799420 d^{2} \mu^{3}+106010745600 d_{1}^{2} \mu^{3}- \\
& 104962054740 d \mu^{3}-3087315 d^{5} d_{1} \mu^{3}-14482314 d^{4} d_{1} \mu^{3}+ \\
& 102619440 d^{3} d_{1} \mu^{3}-378249256 d^{2} d_{1} \mu^{3}-514482969 d d_{1} \mu^{3}+ \\
& 16954862694 d_{1} \mu^{3}-161533242780 \mu^{3}+2592000 d^{4} \mu^{2}+ \\
& 2057529600 d_{1}^{4} \mu^{2}-44829000 d^{3} \mu^{2}-7823939200 d_{1}^{3} \mu^{2}+ \\
& 5429876520 d^{2} \mu^{2}+142752174400 d_{1}^{2} \mu^{2}-151547440440 d \mu^{2}- \\
& 8139285 d^{5} d_{1} \mu^{2}+18386676 d^{4} d_{1} \mu^{2}+185103072 d^{3} d_{1} \mu^{2}- \\
& 2136073170 d^{2} d_{1} \mu^{2}+16421485773 d d_{1} \mu^{2}-7595804986 d_{1} \mu^{2}+ \\
& 331097668120 \mu^{2}-2592000 d^{4} \mu+19615500 d^{3} \mu+2390434200 d_{1}^{3} \mu- \\
& 3752713660 d^{2} \mu-45284356800 d_{1}^{2} \mu+37737977620 d \mu- \\
& 7016625 d^{5} d_{1} \mu+65002014 d^{4} d_{1} \mu-225114120 d^{3} d_{1} \mu+ \\
& 205365160 d^{2} d_{1} \mu+2781415637 d d_{1} \mu-37108488186 d_{1} \mu+ \\
& 96344767260 \mu-2592000 d^{4}-4629441600 d_{1}^{4}+44829000 d^{3}+ \\
& 17603863200 d_{1}^{3}-2672382720 d^{2}-106303896000 d_{1}^{2}+ \\
& 59129835240 d-1964655 d^{5} d_{1}+32133024 d^{4} d_{1}-264687192 d^{3} d_{1}+ \\
& 1861002066 d^{2} d_{1}-14052617733 d d_{1}+30430025010 d_{1}- \\
& 154338492240, \\
& \\
&
\end{aligned}
$$

where the period constant $T_{k}$ has been computed modulo the constants $T_{l}=0$ for $l=2, \ldots, k-1$ and modulo a non-zero constant.

The period constants $T_{2}, T_{4}$ and $T_{6}$ are polynomials in the variables $d, d_{1}$ and $\mu$. We want to study the zeros $\left(d, d_{1}, \mu\right)$ of $T_{2}, T_{4}$ and $T_{6}$ with $d \geq 7$ an odd positive integer. For doing that we consider the resultant of $T_{2}$ and $T_{4}$ with respect to $\mu$. This resultant is a polynomial $f_{1}$ in the variables $d$ and $d_{1}$. After we consider the resultant of $T_{2}$ and $T_{5}$ with respect to $\mu$. This resultant is a polynomial $f_{2}$ in the variables $d$ and $d_{1}$. The polynomials $f_{1}$ 
and $f_{2}$ have in common the factors $d_{1}$. We define the polynomials $g_{1}$ and $g_{2}$ as the polynomials $f_{1}$ and $f_{2}$ omitting the common factor $d_{1}$. Then we consider the resultant of $g_{1}$ and $g_{2}$ with respect to $d_{1}$. This resultant is a polynomial $h$ in the variable $d$. It easy to check that the the polynomial $h$ has no odd positive integers roots $d \geq 7$. In short the common zeros $\left(d, d_{1}, \mu\right)$ of $T_{2}, T_{4}$ and $T_{6}$ must have either $d_{1}=0$.

Assume $d_{1}=0$. Then $T_{2}=5(1-\mu)(d-3+(d+1) \mu)$ and $T_{2}$ divides $T_{4}$ and $T_{5}$. So $d_{1}=0$ and either $\mu=1$ or $\mu=(3-d) /(d+1)$ vanish $T_{2}, T_{4}$ and $T_{5}$. The case $d_{1}=0$ and $\mu=1$ corresponds to the condition (b.1) of Theorem 1. The case $d_{1}=0$ and $\mu=(3-d) /(d+1)$ corresponds to the condition (b.2) of Theorem 1. Hence these two conditions are necessary for having an isochronous center. This completes the proof of Theorem 1(b) when $j=1$.

\section{ACKNOWLEDGEMENTS}

The first author has been supported by the grants MCYT/FEDER Spain MTM2008-03437 and CIRIT-Spain 2005SGR 00550. The second author has been partially supported by FCT through CAMGSD, Lisbon.

\section{REFERENCES}

1. A.A. Andronov, E.A. Leontovich, I.I. Gordon And A.G. Maier, Theory of bifurcations of dynamic systems on a plane, John Wiley and Sons, Nova York-Toronto, 1973.

2. N.N. BAutin, On the number of limit cycles which appear with the variation of coefficients from an equilibrium position of focus or center type, Mat. Sbornik 30 (1952), 181-196, Amer. Math. Soc. Transl. Vol. 100 (1954), 1-19.

3. J. Chavarriga and M. Sabatini, A survey of isochronous centers, Qual. Theory Dyn. Syst. 1 (1999), 1-70.

4. E.S. Cheb-Terrab And A.D. Roche, An Abel ODE class generalizing known integrable classes, European J. of Appl. Math. 14 (2003), 217-229.

5. A. Cima, A. Gasull, V. Mañosa And F. Mañosas, Algebraic properties of the Liapunov and period constants, Rocky Mountain J. Math. 27 (1997), 471-501.

6. H. Dulac, Détermination et integration d'une certaine classe d'équations différentielle ayant par point singulier un centre, Bull. Sci. Math. Sér. (2) 32 (1908), 230-252.

7. A. Gasull, A. Guillamon And V. Mañosa, Centre and isochronicity conditions for systems with homogeneous nonlinearities, in 2nd Catalan Days on Applied Mathematics (Odeillo, 1995), pp. 105116, Presses Univ. Perpignan, Perpignan, 1995.

8. A. Gasull And J. Llibre, Limit cycles for a class of Abel equations, SIAM J. Math. Anal. 21 (1990), 1235-1244.

9. E. KAMKe, Differentialgleichungen "losungsmethoden und losungen", Col. Mathematik und ihre anwendungen, 18, Akademische Verlagsgesellschaft Becker und Erler Kom-Ges., Leipzig, 1943.

10. W. KAPTEYN, On the midpoints of integral curves of differential equations of the first degree, Nederl. Akad. Wetensch. Verslag. Afd. Natuurk. Konikl. Nederland (1911), 1446-1457 (Dutch).

11. W. KAPtEYN, New investigations on the midpoints of integrals of differential equations of the first degree, Nederl. Akad. Wetensch. Verslag Afd. Natuurk. 20 (1912), 13541365; 21, 27-33 (Dutch). 
12. A.M. Liapunov, Problème général de la stabilité du mouvement, Ann. of Math. Studies 17, Princeton Univ. Press, 1949.

13. N. G. LlOYD, Small amplitude lmit cycles of polynomial differential equations, Lect. Notes in Math. 1032 (1983), pp. 346-356.

14. W.S. Loud, Behavior of the period of solutions of certain plane autonomous systems near centres, Contributions to Differential Equations 3, (1964), 323-336.

15. J.M. Pearson, N.G. Lloyd and C.J. Christopher, Algorithmic derivation of centre conditions, SIAM Rev. 38 (1996), 619û-636.

16. I. PleshKan, A new method for investigating the isochronicity of a system of two differential equations, Differential equations 5 (1969), 796-802.

17. H. Poincaré, Mémoire sur les courbes définies par les équations différentielles, Oeuvreus de Henri Poincaré, Vol. I, Gauthiers-Villars, Paris, 1951, pp. 95-114.

18. K. S. SibirSkiI, On the number of limit cycles in the neighborhood of a singular point, Differential Equations 1 (1965), 36-40.

19. H. Zoladek, Quadratic systems with center and their perturbations, J. Differential Equations 109 (1994), 223-273.

20. H. ZOLADEK, On a certain generalization of Bautin's theorem, Nonlinearity 7 (1994), $273-279$.

${ }^{1}$ Departament de Matemàtiques, Universitat Autònoma de Barcelona, 08193 Bellaterra, Barcelona, Catalonia, Spain

E-mail address: jllibre@mat.uab.cat

2 Departamento de Matemática, Instituto Superior Técnico, Av. Rovisco Pais 1049-001, Lisboa, Portugal

E-mail address: cvalls@math.ist.utl.pt 